\title{
Integrating immune checkpoint inhibitors and targeted therapies in the treatment of early stage non-small cell lung cancer: a narrative review
}

\author{
Ana Ortega-Franco ${ }^{1} \wedge, V_{i r g i n i a}$ Calvo $^{2}$, Fabio Franco $^{2}$, Mariano Provencio $^{2} \wedge$, Raffaele Califano $^{1,3,4} \wedge$ \\ ${ }^{1}$ Department of Medical Oncology, The Christie NHS Foundation Trust, Manchester, UK; ${ }^{2}$ Department of Medical Oncology, Hospital \\ Universitario Puerta de Hierro, Madrid, Spain; ${ }^{3}$ Department of Medical Oncology, Manchester University NHS Foundation Trust, Manchester, UK; \\ ${ }^{4}$ Division of Cancer Sciences, University of Manchester, Manchester, UK \\ Contributions: (I) Conception and design: All authors; (II) Administrative support: None; (III) Provision of study materials or patients: None; (IV) \\ Collection and assembly of data: All authors; (V) Data analysis and interpretation: All authors; (VI) Manuscript writing: All authors; (VII) Final \\ approval of manuscript: All authors. \\ Correspondence to: Dr. Raffaele Califano. Department of Medical Oncology, The Christie NHS Foundation Trust, 550 Wilmslow Road, Manchester \\ M20 4BX, UK. Email: raffaele.califano@nhs.net.
}

\begin{abstract}
Prognosis of early stage non-small cell lung cancer (eNSCLC) is poor even when treated radically with surgery and (neo)adjuvant chemotherapy (Cht). The discovery of tyrosine kinase inhibitors (TKIs) for oncogene addicted NSCLC and immune checkpoint inhibitors (ICIs) have revolutionised the therapeutic paradigm and improved survival of advanced NSCLC. The unprecedented impact of these drugs has shifted the focus of investigation to early stage disease aiming at improving cure. In this context, several single arm phase II studies evaluating neoadjuvant ICI alone or in combination with platinum-based Cht have shown encouraging rates of pathological response which have spurred several ongoing randomized trials with (neo)adjuvant ICI. More recently, ADAURA study evaluating adjuvant osimertinib demonstrated a profound reduction of the risk of recurrence in patients with stage I $(>4 \mathrm{~cm})-$ IIIA eNSCLC harbouring EGFR sensitizing mutations. ICIs and TKIs represent a true revolution in the treatment of eNSCLC call to challenge the current standard of care. However, questions regarding drug resistance, recurrence patterns, biomarker identification, optimal treatment duration and sequencing need be answered to effectively integrate new drugs in the rapidly evolving therapeutic landscape of NSCLC. In this review we critically review new developments and future perspectives of TKIs and ICI as (neo)adjuvant strategies for eNSCLC.
\end{abstract}

Keywords: Adjuvant drug therapy; immunotherapy; osimertinib; neoadjuvant therapy; non-small cell lung cancer (NSCLC); targeted molecular therapy

Submitted Apr 16, 2020. Accepted for publication Oct 12, 2020.

doi: $10.21037 /$ tlcr-20-546

View this article at: http://dx.doi.org/10.21037/tlcr-20-546

\section{Introduction}

Lung cancer is the leading cause of cancer mortality worldwide (1). Despite surgery with or without (neo) adjuvant chemotherapy (Cht), the 5-year overall survival
(OS) for resected early stage non-small cell lung cancer (eNSCLC) cancer remains poor and ranges from $25 \%$ to $75 \%$ (2). A high proportion of resected eNSCLC patients will die from metastatic disease indicating that, micro-

^ ORCID: Ana Ortega-Franco, 0000-0002-2865-1855; Mariano Provencio, 0000-0002-2826-656X; Raffaele Califano, 0000-0003-26115319. 
metastasis are present at early stage and remain untreated with surgery. In the last 20 years, several trials have demonstrated that (neo)adjuvant Cht offers a small but consistent benefit in OS over surgery alone and has become standard of care (3).

In the metastatic setting, tyrosine kinase inhibitors (TKIs) for oncogene-addicted tumours and immune checkpoint inhibitors (ICIs) have revolutionised the treatment paradigm of metastatic NSCLC (4). The unprecedented activity of these drugs has shifted the focus of investigation to early stage disease aiming to improve cure rate. In this review we summarize new developments on TKIs and ICIs as novel (neo)adjuvant agents for the treatment of eNSCLC.

For the clarity of this review, it is important to note that all the trials described here use the $7^{\text {th }}$ or earlier editions of the TNM in Lung Cancer issued by International Association for the Study of Lung Cancer (IASLC) which have several differences compared to the most updated $8^{\text {th }}$ edition. Notably, tumours with diameter $>4 \mathrm{~cm}$ were staged as T2N0 (IB $>4 \mathrm{~cm}$ ) in the $7^{\text {th }}$ edition which corresponds to T2bN0 (stage IIA) in the $8^{\text {th }}$ edition. In order to maintain coherence, we will refer to them as IB $>4 \mathrm{~cm}$ or tumours $>4 \mathrm{~cm}$. We present the following article in accordance with the Narrative Review reporting checklist (available at http:// dx.doi.org/10.21037/tlcr-20-546).

\section{Cht in eNSCLC: state of the art}

\section{Adjuvant Cht (A-Cht)}

A-Cht for resected eNSCLC increases 5-year OS by 4-5\% at 5 years (5). This benefit is clear in patients with lymph node involvement (stage II and III) whereas the evidence for node negative (N0) patients is less obvious. A-Cht seems to be detrimental in stage IA and the benefit for stage IB seems to be restricted to tumours with a diameter $>4 \mathrm{~cm}$ $(6,7)$. LACE (8) is a meta-analysis which included over 4,500 patients with resected eNSCLC treated with cisplatinbased A-Cht in five clinical trials. The hazard ratio (HR) for death was 0.89 [95\% confidence interval (CI): 0.82-0.96; $\mathrm{P}=0.005]$ and the absolute benefit in 5 -year OS was $5.4 \%$. Patients with stage II and III derived the greatest benefit whereas A-Cht resulted detrimental for stage IA. Multiple cisplatin-based regimens are accepted but the combination with vinorelbine is the most extensively studied regimen $(9,10)$. In a prespecified subgroup analyses of LACE meta-analysis, the cisplatin-vinorelbine regimen (LACEvinorelbine) demonstrated a superior survival benefit and was recommended in completely resected stages II and III NSCLC (11). The LACE-vinorelbine cohort included 1,888 patients, baseline characteristics were similar to other platinum regimens (LACE-other) but had fewer patients with stage IA ( $2 \%$ vs. $11 \%)$. Survival improvement at 5 years was $8.9 \%$ with LACE-vinorelbine $v$ s. observation (HR: 0.80, 95\% CI: 0.70-0.91, $\mathrm{P}<0.001)$. Stage was a significant predictor for survival $(\mathrm{P}=0.02)$, benefit at 5 years for stage I, II and III was $1.8 \%, 11.6 \%$ and $14.7 \%$, respectively. The overall result of LACE-vinorelbine was statistically superior to LACE-others (HR: 0.95, 95\% CI: 0.86-1.05; $\mathrm{P}=0.04)$. Carboplatin-based Cht can be considered for stage IB patients not eligible for cisplatin (6). The recommended interval from surgery to Cht initiation is around 6 weeks but longer periods do not compromise Cht benefit according to recent data (10).

In summary, A-Cht is recommended in node positive patients and can be considered in node negative with tumours $>4 \mathrm{~cm}$. Cisplatin-vinorelbine is the most commonly used regimen although other platinum-based combinations are accepted (3).

\section{Neoadjuvant Cht (N-Cht)}

Encouraging results from adjuvant trials led to the planning of studies evaluating N-Cht vs. surgery alone. N-Cht has several theoretical advantages: assessment of response to Cht in vivo, improve loco-regional drug delivery due to intact vessels pre-surgery, better tolerability, early treatment of micro-metastatic disease and tumour down-staging with improved resectability. Moreover, N-Cht offers the possibility of evaluating clinical and biological markers, which may serve as surrogates of response and predict longterm outcome. However, N-Cht has potential disadvantages including delay in delivery of local therapy due to toxicity, risk of progression in chemo-resistant patients and higher risk of peri-operative complications.

Notably, many studies designed with surgery alone as control arm were stopped early due to the positive results of A-Cht studies.

Gilligan et al. conducted a multicentre phase III study to assess the benefit of adding N-Cht to surgery in eNSCLC (12). Five hundred and ten patients with stage I to III were randomized to surgery vs. N-Cht followed by surgery. Primary endpoint was OS. N-Cht was feasible ( $75 \%$ of the patients received three cycles) and postoperative complications were similar between groups. Thirty-one percent of patients treated with 
$\mathrm{N}$-Cht had tumour down-staging but the type (lobectomy $v s$. pneumonectomy) or completeness (R0 vs. R1-R2) of surgery was not altered. The addition of Cht did not translate in longer OS, however, the addition of this results to a systematic review of $\mathrm{N}$-Cht of 1,507 patients resulted in an equivalent improvement of $5 \%$ in 5 -year OS (12).

In two other meta-analyses $(13,14), \mathrm{N}$-Cht showed an absolute improvement in 5 -year OS of $5 \%$ to $6 \%$ in patients with stage IB-IIIA disease. The NATCH trial (15) randomly assigned patients with stage I-II to three arms: surgery alone or $\mathrm{N}$-Cht with carboplatin and paclitaxel followed by surgery, or to surgery followed by A-Cht with carboplatin and paclitaxel. The study failed to demonstrate improvement in OS with either strategy $v s$. surgery alone. However, $70 \%$ of randomized patients had stage I disease which do not seem to benefit from A-Cht. Furthermore, the study reported a $5.7 \%$ postoperative mortality after a lobectomy-bilobectomy which is higher than expected. Notably this study captured mortality beyond the standard 30 days postoperative period. Other limitations of the $\mathrm{NATCH}$ trial were that staging with PET/CT scan was not mandatory at study entry and mediastinoscopy was performed in only $12.6 \%$ of patients.

Lim et al. (16) conducted an indirect meta-analysis to compare A-Cht vs. N-Cht. Data was extracted from 10,000 patients included in 32 trials (22 adjuvant trials and 10 neoadjuvant trials). Noteworthy, none of the trials included a head to head comparison between the two strategies. Using indirect comparison analysis, the HRs for OS (HR: 0.80 and HR: 0.81) and disease-free survival (DFS) (HR: 0.76 and HR: 0.79 ) were similar between the A-Cht and $\mathrm{N}$-Cht groups.

\section{Major pathological response (MPR) as a surrogate endpoint}

The time from patient enrolment to data publication in (neo)adjuvant NSCLC trials ranges from 9 to 13 years thus, upon study completion the therapy might be obsolete and the findings irrelevant. As such, there is a need for surrogate markers of efficacy aside from the traditional endpoints of OS or DFS to accelerate the development of new therapies. Complete pathological response (pCR) and MPR are surrogates endpoint frequently used in neoadjuvant trials as these require shorter follow-up than DFS or OS.

The median frequency of pCR with N-Cht in NSCLC is thought to be low, 4\% (0-16\%) (17). However, pCR rates differ significantly between trials as pCR definition and reporting methods are not homogeneous. Some trials report the frequency of $\mathrm{pCR}$ as the fraction of patients surgically explored or completely resected while other consider all patients receiving $\mathrm{N}$-Cht. Most trials define pCR as complete eradication of cancer cells in the primary tumour and lymph nodes while others omit the latter. Only a few trials have prospectively demonstrated the association of pCR with improved survival (18-20) possibly due to the rarity of this event which requires large number of patients to demonstrate survival benefit. The infrequency of pCR limits its adoption as a feasible surrogate biomarker, thus laxer definitions of pathological response have been explored. Junker et al. (21) evaluated the feasibility of morphologic regression grading in 54 patients with eNSCLC treated with neoadjuvant chemo-radiotherapy. Of the 40 resected patients, $37 \%$ had regression grade IIb (defined as less than $10 \%$ of vital tumour in primary lesion and/or mediastinal lymph nodes presenting focal microscopic disease-later defined as MPR) and $12 \%$ had grade III (complete tumour regression in primary tumour and lymph nodes). Patients with regression IIb and III had significantly longer OS (3-year OS: $52 \%$ vs. $9 \%, \mathrm{P}=0.02$ ) than those with lower regression grades. In another study, evaluating 192 patients with eNSCLCs treated with N-Cht and surgery, 5-year DFS and OS were longer in $36(19 \%)$ patients with $\leq 10 \%$ of viable tumour cells (TCs) in resected specimen. Notably, 5 -year OS rate was $85 \%$ vs. $40 \%$ $(\mathrm{P}<0.0001)$ and $\mathrm{DFS}$ was $78 \%$ vs. $35 \%(\mathrm{P}<0.001)$ in $\leq 10 \%$ $v s .>10 \%$ viable TCs, respectively (22).

More recently, Hellmann et al. (17) proposed the use of MPR as a surrogate endpoint for OS in neoadjuvant trials for resectable NSCLC as it strongly correlates with OS and captures the magnitude of the treatment benefit. MPR has been extensively used in neoadjuvant trials with ICIs and TKIs although the application and extrapolation to these therapies has not been validated. Modified pathological criteria of response to ICIs are currently being assessed (23).

\section{(Neo)adjuvant ICls}

The programed cell death ligand 1 (PD-1/PD-L1) and cytotoxic T lymphocyte-associated protein 4 (CTLA-4) axis has been extensively studied for therapeutic checkpoint inhibition in NSCLC. At present, first-line ICI options in metastatic NSCLC include: pembrolizumab monotherapy (24), pembrolizumab in combination with platinum-based Cht $(25,26)$ and atezolizumab in combination with bevacizumab and carboplatin/ 
paclitaxel (27). The ICI doublet with nivolumab plus ipilimumab has also been approved by the Food and Drug Administration (FDA) as first line treatment in patients with PD-L $1 \geq 1 \%$ (28). In addition, CheckMate9LA study with limited Cht plus nivolumab/ipilimumab show significant OS improvement in a pre-planned interim analysis presented in the 2020 American Society of Clinical Oncology (ASCO) meeting (29). A recent metaanalysis including over 4,600 patients from 8 randomized trials showed that ICI plus Cht was associated with improved survival compared to Cht alone as first-line treatment for metastatic NSCLC (HR: 0.74, 95\% CI: 0.64-0.87; $\mathrm{P}=0.0002$ ) (30). Pembrolizumab, atezolizumab and nivolumab have also shown better survival and toxicity profile when compared to docetaxel in pretreated advanced NSCLC patients (31-34). Based on the results of the PACIFIC trial (35), durvalumab is approved as consolidation therapy following concurrent Cht and radiotherapy in non-resectable stage III NSCLC.

Durable tumour response and unprecedented long-term survival rates seen with ICI in selected patients $(36,37)$, together with the success of adjuvant ICI in other solid tumours (38) provides a strong rationale to evaluate ICI in resected eNSCLC.

\section{Neoadjuvant ICIs}

ICIs have been evaluated in the neoadjuvant setting as monotherapy treatment, in combination with Cht and as ICI doublet (Table 1). Despite encouraging results from several small studies, its use remains investigational.

\section{ICI monotherapy}

Forde et al. (39) evaluated the feasibility of neoadjuvant nivolumab in a pilot study with 21 patients with stage (IIIIA) eNSCLC. Two preoperative doses of nivolumab ( $3 \mathrm{mg} / \mathrm{kg}$ ) were given every 2 weeks with planned surgery 4 weeks after the first dose. The primary outcomes were feasibility and safety. Twenty out of 21 (95\%) patients that underwent surgery achieved a complete tumour resection. One patient with grade 3 pneumonia underwent an uncomplicated surgical resection after one dose of nivolumab. There was no delay to lung surgery (median time was 18 days) and no excess of postoperative complications. Treatment-related adverse events (AEs) of any grade occurred in 5/22 (23\%) of patients and only one $\mathrm{AE}$ was grade $\geq 3$. While only $10 \%$ of patients had objective response on post-treatment computed tomography (CT),
MPR occurred in $9(45 \%)$ patients and $3(13 \%)$ patients had pCR (although one of them had residual tumour in hilar node). Two patients in whose tumours had increased in size on pre-surgical CT were found to have minimal or no residual tumour in the surgical specimen. MPR was observed in tumours with higher tumour mutational burden (TMB) and lacking the expression of PD-L1 in TCs but in which infiltrating immune cells (ICs) were highly positive for PD-L1. In one patient with MPR, the immunofluorescence staining of the resection specimen showed a new large influx of CD8 positive T lymphocytes and PD-L1-positive IC into the tumour. Moreover, PD-1 blockade promoted systemic expansion of T-cell clones which shared similar T-cell repertoire with T-cells found in tissue, potentially eliminating microscopic metastatic disease. In the updated analysis with a follow up of 30 months, median RFS has not been reached and the 24 months RFS rate was 69\% (95\% CI: 51-93\%) (45). The presence of circulating DNA (ctDNA) at diagnosis and MPR was not associated with RFS. All patients who had $\geq 30 \%$ reduction in viable tumour after nivolumab demonstrated clearance of detectable ctDNA from blood prior to surgery. Patients with MPR experienced expansion of neoantigen-specific T-cells in peripheral blood. In one patient with ongoing disease-free status, expansion of tumour-associated T-cells has persisted in peripheral blood beyond 15 months from surgery. By contrast, in a patient who had detectable perioperative ctDNA and $75 \%$ residual disease at surgery, minimal T-cell expansion was observed in peripheral blood, with a decreasing frequency of expanded T-cell clones over time that correlated with eventual cancer relapse.

LCMC3 (40) is an ongoing phase II single-arm study of neoadjuvant atezolizumab (two cycles) in patients with resectable stage IB-IIIB (T3N2) eNSCLC. Patients who derived benefit will receive adjuvant atezolizumab for 12 months. The primary endpoint is MPR and planned recruitment is 180 patients. Interim results were presented at the 2019 ASCO congress: 101 patients were included in the safety analysis (90 patients underwent surgery and 11 were not eligible) and efficacy analysis included 77 patients. MPR was reported in 15 (19\%) patients and 4 (5\%) patients had pCR. Six (8\%) patients had partial response (PR), 72 (93\%) had stable disease (SD) and $4(5 \%)$ had progressive disease $(\mathrm{PD})$. There were two treatment-unrelated grade 5 AEs (one cardiac death postsurgical resection, and one death due to PD), 29 grade 3-4 AEs (6\% treatment related). MPR rates correlated with high PD-L1 tumour proportion 
Table 1 Clinical trials evaluating the use of neoadjuvant ICIs in completely resected eNSCLC

\begin{tabular}{|c|c|c|c|c|c|c|c|c|c|c|c|c|}
\hline Trial number/name & Stage & Phase & Experimental treatment & Comparator & $\mathrm{N}$ & $\begin{array}{l}\text { Resected } \\
\text { patients }\end{array}$ & Primary endpoint & pCR [\%] & MPR [\%] & PD [\%] & $A E s \geq 3[\%]$ & Completion date \\
\hline \multicolumn{13}{|l|}{ ICI monotherapy } \\
\hline NCT02259621 (39) & IIIIA & " & Nivolumab $\times 2 \mathrm{C} \rightarrow$ surgery & - & 21 & 20 & $\begin{array}{l}\text { Safety and } \\
\text { feasibility }\end{array}$ & $3^{\dagger}[13]$ & $9[45]$ & $1[5]$ & $1[5]$ & Completed \\
\hline ChiCTR-OIC-17013726 (41) & IB-IIIA & IB & Sintilimab $\times 2 C \rightarrow$ surgery & - & 40 & 37 & Safety & $6[16]$ & $15[41]$ & $4[10]$ & $4[10]$ & Completed \\
\hline \multicolumn{13}{|l|}{ ICI doublet } \\
\hline NCT03158129, NEOSTAR (42) & I-IIIA (single N2) & ॥ & $\begin{array}{l}\text { Nivolumab }(\mathrm{N}) \times 3 \mathrm{C} \rightarrow \text { surgery; nivolumab } \times 3 \mathrm{C}+\text { ipilimumab } \\
\times 1 \mathrm{C}(\mathrm{NII}) \rightarrow \text { surgery }\end{array}$ & - & $\begin{array}{c}44 \\
(23 \mathrm{~N}, 21 \mathrm{NI})\end{array}$ & 34 & MPR & $\begin{array}{c}6[15] \\
(2 \mathrm{~N}, 4 \mathrm{NI})\end{array}$ & $\begin{aligned} 10[24] \\
(4 \mathrm{~N}, 6 \mathrm{NI})\end{aligned}$ & $\begin{array}{c}6[14] \\
(3 \mathrm{~N}, 3 \mathrm{NI})\end{array}$ & $\begin{array}{c}5[11] \\
(4 \mathrm{~N}, 1 \mathrm{NI})\end{array}$ & 07/2022 \\
\hline NCT03081689, NADIM (43) & IIIA-N2 & ॥ & Cht + nivolumab $\times 3 \mathrm{C} \rightarrow$ surgery $\rightarrow$ nivolumab $\times 8 \mathrm{mo}$ & - & 46 & 41 & $2 y$-PFS & 24 [71] & 34 [83] & 0 & - & 06/2022 \\
\hline NCT02716038 (44) & IIIB & ॥ & Cht + atezolizumab $\times 4 \mathrm{C} \rightarrow$ surgery & - & 30 & 11 & MPR & 3 [21] & $7[50]$ & 0 & 12 [85] & $12 / 2020$ \\
\hline NCT02572843 & IIIA-N2 & ॥ & Cht $\times 3 \mathrm{C} \rightarrow$ durvalumab $\times 2 \mathrm{C} \rightarrow$ surgery $\rightarrow$ durvalumab $\times 1$ y & - & 68 & & $1 y$-EFS & & & & & 12/2021 \\
\hline NCT03800134, AEGEAN & $\|A-\| B$ & IIII & Durvalumab $+\mathrm{Cht} \rightarrow$ surgery $\rightarrow$ durvaumab & $\begin{array}{l}\text { Cht }+ \text { placebo } \rightarrow \text { surgery } \rightarrow \\
\text { placebo }\end{array}$ & 300 & & MPR, EFS & & & & & 01/2024 \\
\hline NCT03838159, NADIM 2 & IIIA-IIIB (T3N2) & ॥ & Cht + nivolumab $\times 3 \mathrm{C} \rightarrow$ surgery $\rightarrow$ nivolumab $\times 6$ mo & Cht $\times 3 C \rightarrow$ surgery & 90 & & $\mathrm{pCR}$ & & & & & 09/2027 \\
\hline NCT03425643, KEYNOTE-671 & $\begin{array}{l}\| I-I I A-I I B B \\
(\mathrm{~T} 3-4 \mathrm{~N} 2)\end{array}$ & IIII & $\begin{array}{l}\text { Cht }+ \text { pembrolizumab } \times 4 \mathrm{C} \rightarrow \text { surgery } \rightarrow \\
\text { pembrolizumab } \times 13 \mathrm{C}\end{array}$ & $\begin{aligned} \text { Cht }+ \text { placebo } \times 4 \mathrm{C} \rightarrow \text { surgery } \\
\quad \rightarrow \text { placebo } \times 13 \mathrm{C}\end{aligned}$ & 786 & & EFS, OS & & & & & $06 / 2026$ \\
\hline NCT02998528, Checkmate 816 & IB-IIIA & III & $\begin{array}{l}\text { Cht + nivolumab + ipilimumab } \rightarrow \text { surgery; } \\
\text { Cht + nivolumab } \rightarrow \text { surgery }\end{array}$ & Cht $\rightarrow$ surgery & 350 & & EFS, pCR & & & & & $11 / 2028$ \\
\hline NCT03456063, IMpower 030 & II-IIIA-IIIB (T3N2) & IIII & Atezolizumab + Cht $\times 4 \mathrm{C} \rightarrow$ surgery $\rightarrow$ atezolizumab $\times 16 \mathrm{C}$ & Cht + placebo $\times 4 \mathrm{C} \rightarrow$ surgery & 374 & & MPR, EFS & & & & & 11/2024 \\
\hline NCT04025879, Checkmate $77 \mathrm{~T}$ & $\| I I I I B$ & IIII & Cht + nivolumab $\rightarrow$ surgery $\rightarrow$ nivolumab & $\begin{array}{l}\text { Cht }+ \text { placebo } \rightarrow \text { surgery } \rightarrow \\
\text { placebo }\end{array}$ & 452 & & EFS & & & & & 09/2024 \\
\hline
\end{tabular}

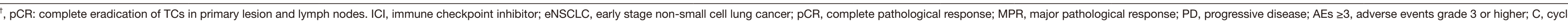
N, estimated enrollment; mo, months; EFS, event-free survival; PFS, progression-free survival; OS, overall survival; Cht, chemotherapy; $y$ year(s); TC, tumour cell. 
score (TPS) assessed by PD-L1 clone $22 \mathrm{C} 3$ [11\% vs. $35 \%$ for TPS $<50 \%$ and TPS $\geq 50 \%$, respectively $(\mathrm{P}=0.040)]$. However, MPR did not correlate with TMB.

Sintilimab (PD-1 inhibitor) was evaluated in a phase IB study enrolling patients with stage IB-IIIA eNSCLC (41). Forty patients received two preoperative cycles of sintilimab followed by surgery. Primary endpoint was safety. Four (10\%) patients experienced grade 3-4 AEs and one patient had grade 5 (pneumonitis). Overall response rate (ORR) was $20 \%, 15(41 \%)$ patients achieved MPR including 6 $(16 \%)$ with pCR (three of them both in primary tumour and lymph nodes). Squamous cell histology had higher MPR rate $(48.4 \%$ vs. $0 \%)$ compared to adenocarcinoma. Notably decrease of maximum standardized uptake values (SUVmax) after sintilimab treatment correlated with pathological remission $(\mathrm{P}<0.00001)$. Baseline $\mathrm{PD}-\mathrm{L} 1$ expression in IC instead of TC correlated with pathological regression $(\mathrm{P}=0.0471)$.

\section{ICI doublet}

In the phase II study NEOSTAR (42), 44 patients with stage I-IIIA (single N2 station) eNSCLC were randomized to three doses of nivolumab $3 \mathrm{mg} / \mathrm{kg}$ (N, 23 pts) or nivolumab plus one dose of ipilimumab $1 \mathrm{mg} / \mathrm{kg}$ (NI, $21 \mathrm{pts}$ ) followed by surgery. Primary endpoint was MPR. Thirty-four patients underwent surgery, seven patients were not resected and three ongoing. MPR occurred in 10/41 (24\%) patients (four and six patients in $\mathrm{N}$ and NI, respectively), pCR in $6(15 \%)$ patients (two in $\mathrm{N}$ and four in NI). Median \% of viable tumour was numerically lower in the NI group $(65 \%$ vs. $20 \%, \mathrm{P}=0.097)$. ORR was $22 \%$. NI induced a higher number of tumour infiltrating lymphocytes compared to N. Pre-treatment tumour PD-L1 TPS was higher in responders (MPR + ORR) vs. non-responders $(80 \%$ vs. $1 \%$, $\mathrm{P}=0.024)$ and the percentage of viable tumour was lower in tumours with PDL-1 TPS $>1 \%$ vs. $\leq 1 \%$ ( $20 \%$ vs. $80 \%$, $\mathrm{P}=0.046$ ).

\section{ICI plus Cht}

NADIM (43) an open-label, single-arm phase 2 trial evaluated the safety and efficacy of N-Cht (paclitaxel + carboplatin, every 3 weeks) plus nivolumab (360 mg IV) for three cycles followed by adjuvant nivolumab $(240 \mathrm{mg}$ IV every 2 weeks for 4 months and $480 \mathrm{mg}$ IV every 4 weeks for 8 months) in 46 patients with resectable stage IIIA (N2 or T4) NSCLC. The primary endpoint was progressionfree survival (PFS) at 24 months. Interim results were presented at 2019 World Lung Cancer Congress
(WLCC), 41 patients underwent complete (R0) surgical resection. None of the patients withdrew from the study preoperatively due to progression or toxicity. In the intention to treat (ITT) analysis, $34(83 \%)$ patients achieved MPR (95\% CI: 68-93\%) and 24 (71\%) achieved pCR (95\% CI: $54-87 \%)$. Down-staging was seen in 38 (90\%) of cases. Twenty-nine (71\%) and 3 (7\%) patients had PR and CR using RECIST criteria, respectively. After a median follow-up of 13.8 months, 12 months PFS was $95.7 \%$ (95\% CI: 84-99\%). Similarly, NADIM 2, an ongoing phase II study will include 90 patients with stage IIIA-IIIB treated with carboplatin/paclitaxel for three cycles alone or with nivolumab followed by adjuvant nivolumab for 6 months. Primary endpoint is pCR.

Atezolizumab was also evaluated in combination with nab-paclitaxel and carboplatin for four cycles prior to surgery in an ongoing phase II study in patients with resectable stage IIIB (T3N2) NSCLC (44). Planned recruitment is 30 patients and primary endpoint is MPR. In the first 14 evaluable patients, PR was $57 \%$ and the remainder had SD. Eleven patients underwent resection successfully and one patient had post-operative complications unrelated to study drugs leading to death. Seven/fourteen (50\%) patients had MPR including 3 (21\%) with pCR. MPR was seen regardless of PD-L1 status. The most common toxicity was neutropenia ( $86 \%$ grade $3-4)$ with 9/14 patients requiring Cht dose reduction.

Another phase II study (NCT02572843) with the PDL1 inhibitor durvalumab will report results in 2021. Patients with stage IIIA(N2) disease will receive 3 cycles of platinumbased Cht followed by 2 cycles of durvalumab prior surgery. Patients with R0 resection will proceed to adjuvant durvalumab for 1 year and patients with R1/R2 disease will receive chest radiotherapy prior adjuvant durvalumab. The primary endpoint is 12-month event-free survival (EFS).

In the next few years, several phase III trials (Table 1) will aim to validate the benefit of adding PD-1/PD-L1 inhibitors to standard $\mathrm{N}$-Cht in large randomized studies. The results from these studies is key to effectively adopt ICIs in the neoadjuvant setting. The KEYNOTE-671, IMpower030, AEGEAN and Checkmate 77T trials have a similar design; patients with stage II to IIIB will receive $\mathrm{N}$-Cht alone or plus placebo vs. N-Cht plus pembrolizumab, atezolizumab, durvalumab or nivolumab, respectively, followed by the correspondent adjuvant therapy or standard of care/placebo. IMpower030, AEGEAN and Checkmate $77 \mathrm{~T}$ primary endpoints are pathological response and EFS. Notably, KEYNOTE-671 is the only trial powered to detect OS 


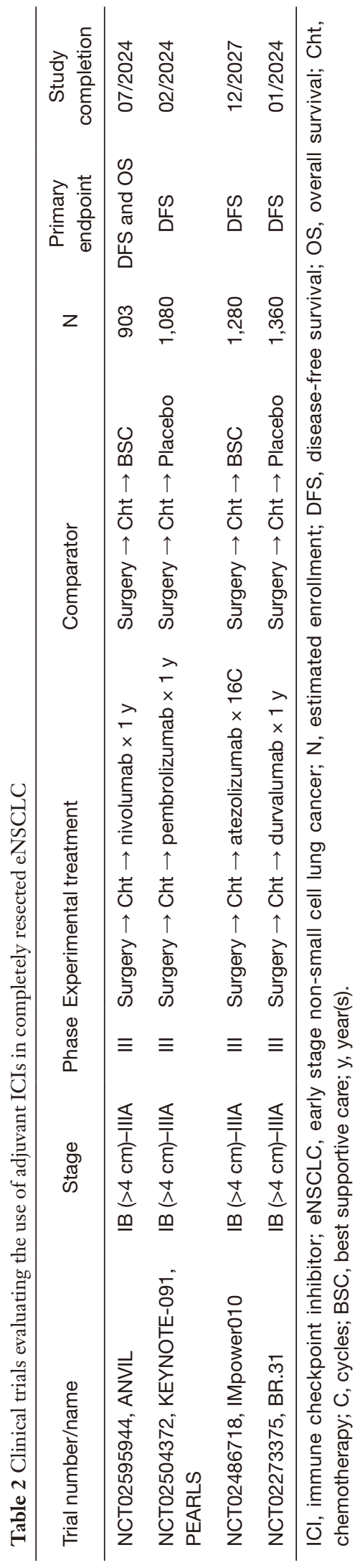

benefit. The Checkmate 816 is a three-arm study that will randomize 350 patients with stage IB-IIIA to receive nivolumab plus $\mathrm{N}$-Cht $v s$. nivolumab plus ipilimumab plus $\mathrm{N}$-Cht vs. N-Cht alone. Adjuvant therapy is not allowed. Co-primary endpoints are pCR and EFS.

\section{Adjuvant ICIs}

Several ongoing trials aim to evaluate the impact of adding ICI to A-Cht in completely resected NSCLC (Table 2). Due to the nature of the disease, results from these trails will be available in several years. ANVIL (nivolumab), PEARLS (pembrolizumab), IMpower010 (atezolizumab) and BR.31 (durvalumab) trials have a similar design. All of them will include tumours stage I $\geq 4 \mathrm{~cm}-\mathrm{IIIA}$ and PD-1/PD-L1 therapy will be administrated for up to 1 year following A-Cht completion. PEARLS and BR.31 are placebo controlled. DFS is the primary endpoint for all trials and only ANVIL study is powered for OS.

\section{(Neo)adjuvant targeted therapies}

Recent advances in personalized medicine have identified oncogene-addicted subgroups of lung cancer amenable to TKIs. The most well studied genetic alterations are those occurring in the epidermal growth factor receptor (EGFR) gene (deletion in exon 19 and point mutation L858R in exon 21), ALK gene rearrangement and ROS protooncogene 1 (ROS1) rearrangement. Taken together, EGFR, ALK and ROS1 molecular aberrations can be detected in about $20 \%$ of lung cancer in western population, mainly in non-smokers patients with adenocarcinoma histology. Additionally, new sequencing techniques have prompted the discovery of other genetic aberrations (in RET, BRAF, MET genes) some of which are already treatable with approved drugs or under investigation. In EGFR and ALK positive patients, TKI have proved to be superior to platinum Cht in terms of PFS and ORR in the metastatic setting (46-48).

The role of oncogenic drivers in the prognosis of resected eNSCLC is debated. Two studies comparing completely resected stage I and I-III NSCLC failed to find differences in DFS and OS between EGFR-wild type (EGFRwt) and EGFR-mutant (EGFRmt) patients (49,50). Similarly, ALK positivity did not predict tumour recurrence after surgery in two retrospective studies $(51,52)$. Conversely, EGFR was found to be associated with more frequent distant relapses and worse 5 -year PFS in a retrospective study of 168 patients with N2 disease treated 
with neoadjuvant chemo-radiotherapy followed by surgical resection (53). Oncogene addiction is a strong predictive factor for TKI response thus TKIs are an attractive strategy for eNSCLC. The vast majority of the reported trials are limited to EGFRmt patients.

\section{Neoadjuvant TKIs}

The first study to demonstrate that EGFR mutations could be used to select patients for neoadjuvant TKI was conducted by Rizvi et al. (54). A cohort of 50 patients with eNSCLC enriched for the presence of EGFRmt ( $\leq 15$ pack-year cigarette smoking history and/or a component of bronchioloalveolar carcinoma), were treated with preoperative gefitinib for 21 days. Twenty-one patients achieved a radiological tumour reduction of $\geq 25 \%, 17$ were EGFRmt and 4 were EGFRwt ( $\mathrm{P}=0.0001)$ (Table 3).

ESTERN (55) was a small, single arm study to test the feasibility of neoadjuvant TKI in stage IIIA-N2 (endobronchial ultrasound confirmed) EGFRmt eNSCLC. Only patients with benefit from neoadjuvant erlotinib underwent surgery. The primary endpoint was resection rate. Twenty-five patients received neoadjuvant erlotinib for 56 days, 16 of them underwent surgery and radical resection rate was $60 \%$. No unexpected toxicities were observed.

The first randomized study (56) to evaluate the role of biomarker-guided neoadjuvant treatment included 24 patients with resectable IIIA-N2 eNSCLCs. Patients were randomized to receive neoadjuvant erlotinib or gemcitabine and carboplatin (GC) based on EGFR status. The primary endpoint of ORR was $58 \%$ in the erlotinib arm in EGFRmt and $25 \%$ in GC arm in EGFRwt $(\mathrm{P}=0.18)$. However, this improvement in ORR did not translate in longer OS. In fact, the most common site of recurrence in patients treated with erlotinib was distant metastases in 10/12 (83\%). In the GC arm, the local and distant recurrence rate were similar $5 / 12(41 \%)$ and $7 / 12(58 \%)$, respectively. Patients in the GC arm had longer PFS (9.0 vs. 6.9 months, $\mathrm{P}=0.071)$ and OS (28.1 vs. 14.5 months, $\mathrm{P}=0.201)$ compared to those in the erlotinib arm. According to the authors, the lack of survival benefit in the erlotinib arm could be due to tumour flare after TKI discontinuation as this phenomenon had been previously described in metastatic EGFRmt (59). The sudden removal of oncogene inhibition upon TKI termination prior surgery could promote potential residual circulating TCs to accelerate and rebound, resulting in more aggressive disease.

CTONG-1103 study (57) is a phase II trial that randomized 72 EGFRmt patients with stage IIIA$\mathrm{N} 2$ to receive neo- and adjuvant erlotinib $(150 \mathrm{mg} / \mathrm{d}$, neoadjuvant therapy for 42 days and adjuvant therapy up to 12 months) or GC Cht (two cycles pre-surgery and up to two cycles post-surgery). The primary endpoint of ORR was not met, ORR was $54 \%$ vs. $34 \%$ for erlotinib and for GC, respectively (odds ratio: 2.26, 95\% CI: 0.87-5.84; $\mathrm{P}=0.092)$. No pCR observed in either arm. Median PFS was significantly longer with erlotinib, $21.5 v s$. GC 11.4 months (HR: 0.39, 95\% CI: 0.23-0.67; $\mathrm{P}<0.001$ ).

Neoadjuvant crizotinib was also evaluated in a small study ( $\mathrm{n}=11)$ in locally advanced (N2 disease) ALK positive patients (58). Ten (91\%) patients achieved a R0 resection and $2(18 \%)$ patients had pCR. Interestingly, 5 of the 6 patients who relapsed, responded to crizotinib re-challenge. An ongoing trial (NCT03088930) is investigating the efficacy of crizotinib in resectable eNSCLCs harbouring crizotinib-sensitive molecular aberrations (ALK, ROS1 or MET exon 14).

The FLAURA trial $(60,61)$ granted FDA and European Medicines Agency (EMA) approval of osimertinib as new standard of care for first line advanced EGFRmt NSCLC. Osimertinib achieved longer PFS (18.9 vs. 10.2 months, HR: 0.46; 95\% CI: 0.37-0.57; $\mathrm{P}<0.001)$ and $\mathrm{OS}(38.6$ vs. 31.8 months, HR: $0.80,95 \%$ CI: 0.64-0.99; $\mathrm{P}=0.0462$ ) compared to first generation TKI. These encouraging results have prompted two ongoing trials with neoadjuvant osimertinib.

\section{Adjuvant targeted therapies}

In the EGFRmt population, several clinical trials have evaluated adjuvant TKI as consolidation treatment following A-Cht or as a substitute for A-Cht (Table 4).

\section{TKI as consolidation therapy following $A-C h t$}

NCIC CTG BR.19 trial (62) aimed at randomizing 1,242 patients with stage IB-IIIA to gefitinib or placebo for 2 years after completing A-Cht. The trial closed prematurely after randomizing 503 patients due to negative results of two other trials with gefitinib $(71,72)$. No benefit was seen in terms of OS and DFS but only $3 \%$ of the trial population was EGFRmt.

RADIANT (63) was a randomized trial in patients with resected IB-IIIA whose tumour expressed EGFR protein by immunohistochemistry or EGFR amplification by fluorescence in situ hybridization (FISH). Nine hundred 
Table 3 Clinical trials evaluating the use of neoadjuvant TKIs in patients with oncogene addicted NSCLC

\begin{tabular}{|c|c|c|c|c|c|c|c|c|c|c|c|c|}
\hline Trial number/name & Stage & Phase & Molecular driver & Experimental treatment & Comparator & $\mathrm{N}$ & Primary endpoint & ORR & DFS (mo) & PD [\%] & $A E s \geq 3[\%]$ & Completion date \\
\hline Rizvi et al. (54) & I-II & II & Enriched for EGFRmt† & $\begin{array}{l}\text { Gefitinib } 21 \text { days } \rightarrow \\
\text { surgery } \rightarrow \text { gefitinib } 2 y \\
\text { (if response) }\end{array}$ & - & 50 & $\begin{array}{l}\text { Correlation between tumour } \\
\text { regression and EGFR status }\end{array}$ & $\begin{array}{l}21(42 \%) \geq 25 \% \text { response, } 17 \text { vs. } 4 \\
\text { EGFRmt vs. EGFRwt }(P=0.0001)\end{array}$ & $\begin{array}{l}2 \mathrm{y} \text {-DFS } 90 \% \text { vs. } 75 \% \\
\text { (EGFRmt vs. EGFRwt) }\end{array}$ & 8 [16] (only 1 EGFRmt) & 1 [2] & Completed \\
\hline NCT01217619, ESTERN (55) & IIIIA-N2 & $\|$ & EGFRmt & Erlotinib $\rightarrow$ surgery & - & 25 & Radical resection rate & $32 \%$ (60\% radical resection) & 10.4 & 6 [24] & 0 & Completed \\
\hline NCT00600587 (56) & IIIA-N2 & "I & EGFRmt and EGFRwt & $\begin{array}{l}\text { Erlotinib } \rightarrow \text { surgery } \\
\text { (EGFRmt) }\end{array}$ & $\begin{array}{l}\text { Cht } \times 3 C \rightarrow \text { surgery } \\
(\text { EGFRwt) }\end{array}$ & 24 & ORR & $58 \%$ vs. $25 \%(P=0.18)$ & $\begin{array}{l}6.0 \text { vs. } 9.0, \text { HR: } 2.26 \\
(P=0.071)\end{array}$ & $\begin{array}{l}\text { Erlotinib: } 3 \text { [25]; Cht: } 2 \\
\text { [17] }\end{array}$ & $2[17]^{+t}$ & Completed \\
\hline $\begin{array}{l}\text { NCT01407822, } \\
\text { CTONG-1103 (57) }\end{array}$ & IIIA-N2 & "I & EGFRmt & $\begin{array}{l}\text { Erlotinib } \rightarrow \text { surgery } \rightarrow \\
\text { erlotinib } 1 \text { y }\end{array}$ & $\begin{array}{l}\text { Cht } \times 2 \mathrm{C} \rightarrow \text { surgery } \rightarrow \text { Cht } \\
\times 2 \mathrm{C}\end{array}$ & 72 & ORR & 54\% vs. 34\%, OR: $2.26(\mathrm{P}=0.092)$ & $\begin{array}{l}21.5 \text { vs. 11.4, HR: } 0.39 \\
(P<0.001)\end{array}$ & $\begin{array}{l}\text { Erlotinib: } 1 \text { [3]; Cht: } 2 \\
\text { [6] }\end{array}$ & 0 & Completed \\
\hline Zhang et al. (58) & IIIA-N2 & II & ALK & Crizotinib $\rightarrow$ surgery & - & 11 & Feasibility & $91 \%$ Ro resection & - & 0 & $1[9]$ & Completed \\
\hline NCT03088930 & I-IIIA & $\|$ & ALK, ROS1, MET & Crizotinib $\rightarrow$ surgery & - & 18 & ORR & & & & & $10 / 2021$ \\
\hline ChiCTR1800016948 & II-IIIA & $\|$ & EGFRmt & Osimertinib $\rightarrow$ surgery & - & 40 & ORR & & & & & \\
\hline NCT03433469 & I-IIIA & ॥ & EGFRmt & Osimertinib $\rightarrow$ surgery & - & 27 & MPR & & & & & 05/2022 \\
\hline
\end{tabular}

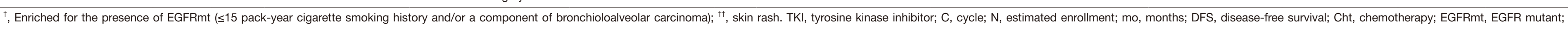

EGFRwt, EGFR wild-type; ORR overall response rate; RO, complete surgical resection: PD, progressive disease; AEs $>3$, adverse events grade 3 or higher; OR, odds ratio; $y$, year(s).

Table 4 Clinical trials evaluating the use of adjuvant TKIs in patients with completely resected oncogene addicted NSCLC

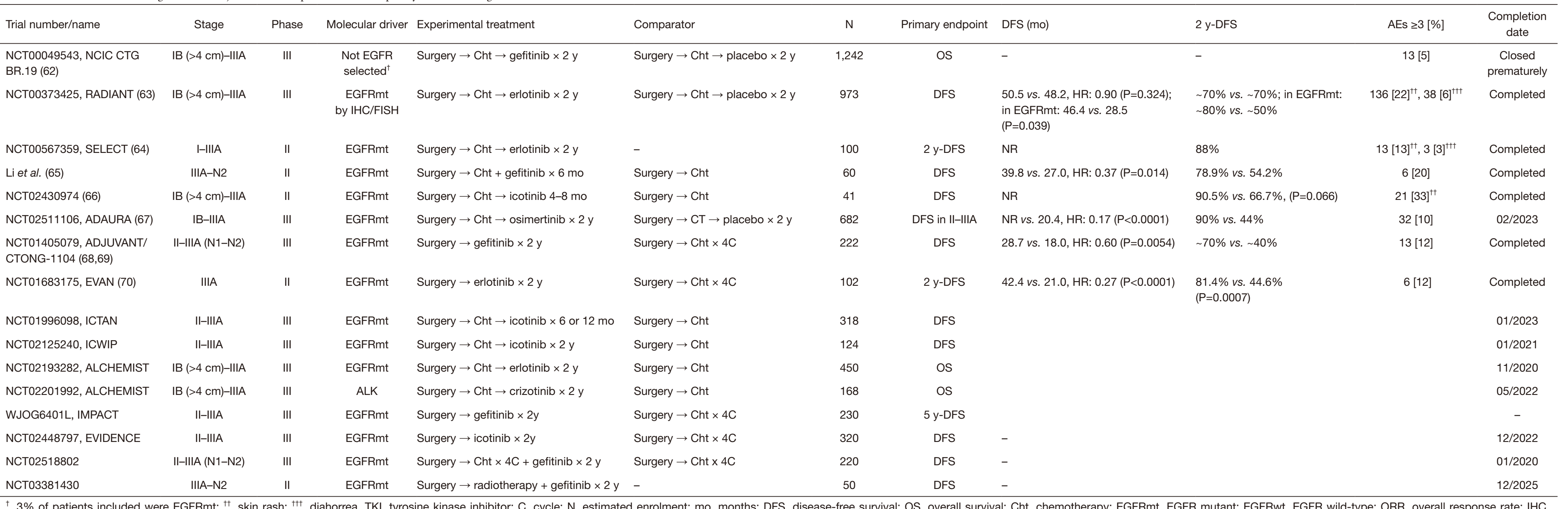

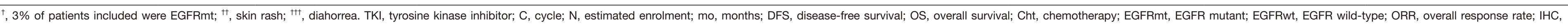
immunochemistry; FISH, fluorescence in situ hybridization; AEs $\geq 3$, adverse events grade 3 or higher; NR, not reached; $y$, year(s). 
and seventy-three patients were randomized 2:1 to receive erlotinib $150 \mathrm{mg}$ once a day or placebo for 2 years after A-Cht. The primary end point of DFS was not met (50.5 vs. 48.2 months, HR: 0.90 , 95\% CI: 0.74-1.10; $\mathrm{P}=0.324$ ), for erlotinib and placebo group, respectively. However, among the 161 (16.5\%) EGFRmt patients, DFS favoured erlotinib (46.4 vs. 28.5 months, HR: 0.61, 95\% CI: 0.38-0.98; $\mathrm{P}=0.039$ ) but this was not statistically significant because of the hierarchical testing procedure. Results from this trial must be interpreted with caution as EGFRmt in the control group underperformed probably due to an imbalance in tumour stage between groups. More importantly, RADIANT trial highlighted that the benefit of adjuvant TKI is restricted to patients harbouring EGFR sensitizing mutations.

More recent trials have incorporated EGFR genotype for patient selection. SELECT (64) trial was a single arm study in resected IA-IIIA EGFRmt NSCLC patients who received adjuvant erlotinib $150 \mathrm{mg}$ /day for 2 years, after standard A-Cht plus/minus thoracic radiotherapy. The study aimed to demonstrate a 2-year DFS greater than $85 \%$. $69 \%$ of the patients completed the planned 2-year course. After a median follow-up of 5.2 years, the 2-year DFS was $88 \%$ and 5 -year OS rate was $86 \%$. Two other small trials $(65,66)$ randomized resected stage IIIA EGFRmt patients to consolidation TKI after platinum-based Cht $v s$. Cht alone. The 2-year DFS was 79-90\% and significantly better in the TKI arm.

To further evaluate the role of adjuvant EGFR-TKI, a meta-analysis including 1,960 patients from five studies (two of them retrospective) was conducted (73). In the EGFRmt population (34\%), EGFR-TKIs were associated to longer DFS (HR: 0.48; 95\% CI: 0.36-0.65) corresponding to an absolute benefit of $9.5 \%$ at 3 years. OS was marginally increased (HR: 0.72, 95\% CI: 0.49-1.06).

ADAURA is a phase III, double-blind, placebocontrolled study to evaluate the efficacy of osimertinib in nonsquamous stage IB-IIIA NSCLCs with EGFR exon 19 deletion or L858R mutation (67). An unplanned interim analysis was presented at the 2020 ASCO meeting after an independent data monitoring committee recommended unblinding of the study due to early efficacy. At the time of the unblinding, the trial had completed enrolment and all patients were followed up for at least 1 year. The trial randomized 682 patients $(1: 1)$ to receive $80 \mathrm{mg}$ of osimertinib or placebo for up to 3 years. Patients had undergone complete resection and had a maximum interval between surgery and randomization of 10 weeks in patients without A-Cht and 26 weeks in patients with A-Cht. Patients' baseline characteristics were well balanced between groups, each tumour stage group (IB/II/III) represented a third of the cohort and half of the patients received A-Cht. With $33 \%$ of data maturity, the primary end point of investigator-assessed DFS in patients with stage IIIIIA disease was met, DFS not reached with osimertinib [38.8-not reached (NR)] vs. 20.4 (16.6-24.5) months with placebo (HR: 0.17, 95\% CI: 0.12-0.23; $\mathrm{P}<0.0001$ ). DFS in stage II-IIIA at 1, 2, and 3 years in osimertinib vs. placebo were $97 \%$ vs. $61 \%, 90 \%$ vs. $44 \%$ and $80 \%$ vs. $28 \%$, respectively. Moreover, DFS in overall population (IBIIIA) (29\% maturity) was also not reached with osimertinib (NR-NR) and $28.1(22.1-35.8)$ months with placebo (HR: $0.21,95 \%$ CI: $0.16-0.28$; $\mathrm{P}<0.0001)$. The DFS benefit was also observed in all subgroups including patient without prior A-Cht. OS data is still immature. Osimertinib was well tolerated and safety profile is consistent with previous data. The median duration of exposure was 22.3 months for osimertinib and 18.4 months for placebo. AEs $\geq 3$ and treatment discontinuation occurred in $20 \%$ and $11 \%$ vs. $14 \%$ and $4 \%$ of patients in the osimertinib and placebo arms, respectively. No treatment related deaths were recorded.

\section{Adjuvant TKI as a substitute of A-Cbt}

In CTONG-1104 (68), 222 patients with completely resected stage II-IIIA EGFRmt NSCLC were randomized 1:1 to receive geftinib for 2 years or cisplatin/vinolrebine for four cycles. The primary endpoint was DFS. All included patients were node positive: N1 (35\%) and N2 $(65 \%)$ disease, balanced between arms. Seventy-two $(68 \%)$ patients received Gefitinib for at least 18 months and 73 (84\%) completed four cycles of Cht. After a median followup of 36.5 months, median DFS in ITT was significantly longer (28.7 vs. 18.0 months, HR: 0.60, 95\% CI: 0.42-0.87; $\mathrm{P}=0.005)$ in the gefitinib arm compared to A-Cht arm. Three-year DFS was 34\% and 27\% (HR: 0.74, 95\% CI: $0.42-1.32 ; \mathrm{P}=0.37)$ in the gefitinib arm and in the A-Cht arm, respectively. Serious AEs (7\% vs. $23 \%$ ) and quality of life was better with gefitinib compared to A-Cht. Final survival analyses were recently presented in the 2020 ASCO Meeting (69). The updated 3-year DFS continues to favour Gefitinib over A-Cht, 39.6\% vs. 23.2\%, (HR: 0.56, 95\% CI: $0.4-0.79 ; \mathrm{P}=0.001)$. However, 5-year DFS is comparable between arms $(22.6 \%$ and $23.2 \%$ in gefitinib vs. A-Cht) which translates into similar survival, mOS of $75.5 \mathrm{vs}$. 62.8 months with gefitinib vs. A-Cht, respectively (HR: 0.92, 
95\% CI: $0.62-1.37 ; \mathrm{P}=0.686)$. The authors also reported data on subsequent lines of treatment, $36.8 \%$ of patients in the gefitinib arm $v s .51 .5 \%$ in the A-Cht arm received subsequent lines of TKI, $31.6 \%$ vs. $22.1 \%$ received other types of treatments and a third of patients in either arm (31.6\% vs. 26.4\%) did not received further treatment. In the gefitinib arm, 9 patients received subsequent osimertinib (ORR: 55.6\%) and 19 patients had other TKI (ORR: 42.1\%). In the Cht arm, 6 patients had osimertinib (ORR: $33.3 \%$ ) and 29 patients had other TKIs (ORR: 29.7\%). Post hoc survival analysis showed that patients receiving adjuvant gefitinib followed by TKI at relapse had significantly longer survival compared to those receiving A-Cht followed by TKI [NR (65.9-NR) vs. 62.8 (40.5-NR) months] or other strategies $(\mathrm{P}<0.001)$. Additionally, the duration of adjuvant gefitinib $\geq 18$ months provided longer survival at relapse, NR (64.0-NR) vs. 35.7 (25.7-NR) months in $\geq 18(\mathrm{n}=72)$ vs. $<18$ months $(\mathrm{n}=18)$, respectively (HR: $0.38,95 \%$ CI: $0.22-0.66 ; \mathrm{P}<0.0001)$.

Similarly, EVAN trial (70) enrolled 102 Chinese patients with completely resected stage IIIA EGFRmt NSCLC. Patients were randomized (1:1) to receive erlotinib 150 $\mathrm{mg}$ daily for up to 2 years or vinorelbine/cisplatin for four cycles. Almost all patients were N2 positive. Thirtynine $(76 \%)$ out of the 51 patients in the erlotinib arm completed at least 18 months of treatment and $32(63 \%)$ patients received four cycles of Cht. After a median followup of 33.0 months, the trial met its primary endpoint demonstrating a higher 2-year DFS rate of $81.4 \%$ in the erlotinib group vs. $44.6 \%$ in the A-Cht group. The difference in 2-year DFS between the groups was $36.7 \%$ (95\% CI: $15.5-58.0 ; \mathrm{P}=0.0007$ ). In a post-hoc analysis for 3 -year DFS, this benefit was sustained (54.2\% vs. 19.8\%). Grade $\geq 3$ AEs rate was $12 \%$ and $26 \%$ in the erlotinib and A-Cht arms, respectively. OS data is immature.

The absolute 3-year DFS difference observed between TKI and Cht arms favours EVAN trial compared to CTONG-1104 (34\% increase vs. $16.4 \%$ increase, respectively). However, this could be due to the fact that EVAN trial included patients with poorer prognosis (higher proportion of N2 disease). Smaller number of patients and wider CIs in EVAN trial might be another explanation.

The unprecedented DFS benefit reported in ADAURA is beyond that seen in any previous trial with (neo) adjuvant EGFR-TKI. More importantly, the DFS curve of osimertinib seems to predict a significant survival advantage thus standard of care for EGFRmt patients with completely resected eNSCLC may change in the next few years. Ongoing adjuvant trials (Table 4) will undoubtedly add valuable clinical data to this scenario, however, it seems unlikely that first generation EGFRTKIs given as a substitute of A-Cht [WJOG6401L (gefitinib) and NCT024488797 (icotinib)] or consolidation after A-Cht [NCT0212540 (icotinib) and NCT02193282 (erlotinib)] will effectively compete with osimertinib. If this is true, these trials may have little margin to impact in clinical practice. In this context, other strategies such as the combination of gefitinib with concurrent A-Cht or radiotherapy (NCT02518802 and NCT03381430) could be more attractive.

\section{Discussion}

The excitement about ICI in resectable eNSCLC started with the striking rates of MPR and pCR reported in a small feasibility study where patients received two cycles of nivolumab ahead of surgery (39), MPR was $45 \%$ and pCR was $13 \%$ which represents a significant improvement from historical data with Cht (17). More recently, another small phase II trial with neoadjuvant Sintilimab showed similar rates of MPR and pCR (41). However, the interim analysis of LCMC3 trial (40), reported more discreet pathological response rates (MPR 19\% and pCR 5\%). Moreover, 11\% of the patients randomized in the trial did not proceed to surgery. Although the causes for this have not been fully explained, PD occurred in $5 \%$ of the patients with one death related to tumour progression. These data are in line with other neoadjuvant trials with ICI monotherapy where PD ranged from $5 \%$ to $10 \%$.

The idea behind combining PD-1/PDL-1 inhibitors with other therapies is to improve pathological response, which in turn reduces the risk of PD prior to surgery. In NEOSTAR (42), the reduction of viable tumour was indeed higher in patients with the doublet nivolumab/ipilimumab compared to nivolumab alone. However, this difference did not reach statistical significance and PD occurred in $15 \%$ of patients. Moreover, there was one death related to a broncopleural fistula due to prolonged steroids for pneumonitis which might question the efficacy and safety of this strategy. The Spanish Lung Cancer Group led the first trial evaluating neoadjuvant platinum-based Cht plus nivolumab followed by adjuvant nivolumab in stage IIIA-N2 (44). Although the primary endpoint of PFS is still immature, the MPR and pCR rates observed are the highest ever reported for a neoadjuvant trial in lung cancer. In addition, none of the patients withdrew from the study preoperatively due 
to PD or toxicity. Preliminary PFS at 12 -month is $98 \%$ which exceeds historical data $(74,75)$. Similarly, a small trial evaluating atezolizumab plus Cht reported favourable but lower rates of pathological response (44), which might reflect poorer prognosis in this cohort (stage IIIB) despite receiving a higher number of cycles of treatment $(4 v s .3)$.

Several ongoing phase III trials in the neoadjuvant setting are focused on Cht plus ICI combinations. Although the backbone therapy is similar for all trials (PD-1 or PDL1 inhibitor plus platinum-based Cht), the number of cycles prior surgery, tumour stage at enrolment and the addition or not of adjuvant ICI, differs between trials. While different approaches might add valuable information to this scenario, the lack of homogeneity in trial design will hamper cross-trial comparison.

To the best of our knowledge, Cht-free options (i.e., ICI as monotherapy or doublet) will not be further explored in upcoming neoadjuvant trials. This is somehow disappointing as the KEYNOTE-024 trial (24) highlighted that Pembrolizumab alone was sufficient to achieve good tumour control in population with $\mathrm{PD}-\mathrm{L} 1 \geq 50 \%$. While this hypothesis might hold true for eNSCLC, the lack of biomarkers that help selecting patients for ICI benefit hinder trial design. Results from correlative analyses conducted in the aforementioned trials are inconsistent possibly due to the scarcity of consensus in the type of assays and methods used to evaluate PD-L1 and TMB, amid the lack of deeper understanding of tumour biology. PD-L1 TPS in IC but not in TC correlated with better pathological regression in trials evaluating neoadjuvant nivolumab and sintilimab. Conversely, in LCMC3 trial, PD-L1 TPS $>50 \%$ significantly correlated with MPR but in NEOSTAR trial, the cut-off was $>1 \%$. Correlative analyses from NADIM trial are not currently available but, in the small cohort reported with atezolizumab plus Cht, MPR occurred regardless of PD-L1 TPS. Interestingly, molecular response data on neoadjuvant nivolumab suggests that ctDNA and peripheral T-cell expansion could be used as potential biomarkers for response and surveillance (45).

The survival benefit of Cht in resectable NSCLC is independent of its use prior to or after surgery. However, pre-clinical models suggest that PD-1 monotherapy is more effective when given as neoadjuvant therapy (76). The biological explanation for this effect possibly lies on the mechanism of action of ICI which, in order to mount a tumour specific immune response, require in situ exposure of ICI with the tumour and its associated specific neoantigens. This phenomenon is consistent with data reported by Forde et al. (39) who found, in patients treated with neoadjuvant PD-1, a systemic expansion of selected T-cells with the potential to treat micro-metastatic disease. How this will effectively impact the outcome of adjuvant ICIs will be revealed in upcoming trials.

At present, the combination of Cht plus ICI given as neoadjuvant treatment represents the most attractive strategy for resectable eNSCLC. However, this area of research is still in early days and results are based in single arm studies with limited number of patients. Data from large randomized trials that validate the safety and efficacy of this strategy not only in terms of MPR but also for EFS and OS is key to move forward. Furthermore, correlative analysis will hopefully help to refine patient selection, understand treatment resistance and potentially identify biomarkers of response and survival.

The vast majority of published studies on (neo) adjuvant targeted therapy have been performed on EGFRmt population hence results from ongoing trials (NCT02201992 and NCT03088930) in less common oncogenic-driven subtypes are awaited with great interest.

One of the concerns with EGFR-TKIs is that they can only suppress rather than eliminate the growth of residual disease which might partially explain the lack of survival benefit observed in prospective trials and retrospective series with (neo)adjuvant EGFR-TKI. However, there are many other explanations that justify this phenomenon such as the size/power of the trials, the lack of comparator arm or inadequate patient selection. More recently, results from the two pivotal adjuvant trials CTONG-1104 and ADAURA point out that the type of TKI (gefitinib, first generation $v$ s. osimertinib, third generation) could be key to attain survival benefit.

In the neoadjuvant setting, EGFR-TKIs achieve higher response rates compared to platinum-based Cht (56). However, neoadjuvant EGFR-TKIs alone can be detrimental in terms of PFS possibly due to tumour flare upon sudden treatment discontinuation (56). Zhong et al. showed that prolonging EGFR inhibition with 1 year of adjuvant TKI (following neoadjuvant TKI and surgery) resulted in longer PFS compared to Cht (57). Although the trial was not powered to detect PFS differences, these results could indicate that the benefit of EGFR-TKI in eNSCLC is driven by prolonged adjuvant EGFR inhibition. Thus, the added benefit of neoadjuvant strategies remains unclear. Two ongoing phase II studies of preoperative 
osimertinib will investigate whether more potent EGFR inhibitors can achieve favourable MPR.

In the adjuvant setting, EGFR-TKIs given as consolidation therapy following A-Cht achieve a 2-year DFS of $80-92 \%$ which seems to be consistent between trials and retrospective series. However, small number of patients $(65,66)$, inappropriate patient selection $(62,63)$ and the lack of comparator arm (64) precluded reaching conclusions to change clinical practice (77). In this context, results from ADAURA and CTONG-1104 are key to understand the role of adjuvant TKI. In ADAURA, the risk of recurrence was reduced by $79 \%$ in patients with stage IB $>4 \mathrm{~cm}-$ IIIA treated with 3 years of adjuvant osimertinib. The DFS benefit was consistent across all subgroups (race, type of EGFR mutation, tumour stage, sex and smoking history) and irrespective of previous A-Cht which could support the use of osimertinib to substitute A-Cht. Importantly, this DFS benefit is also seen in very early stages [patients with node negative disease and tumour diameter $>4 \mathrm{~cm}$ (IB $>4 \mathrm{~cm}$ in TNM $\left.7^{\text {th }}\right)$ ] for whom the role of A-Cht is debated. Although DFS in IB $>4 \mathrm{~cm}$ is a secondary endpoint of the study, a $50 \%$ reduction in the risk of relapse opens the door for osimertinib in this population. The true survival impact of osimertinib is currently unknown, however, the substantial DFS improvement reported in ADAURA may translate in longer survival. In fact, in the 2020 ASCO meeting Dr. Herbst gave an early snapshot of the OS curve which estimates a $7 \%$ absolute increase at 2 years in patients with stage II-IIIA (HR: 0.40, 95\% CI: 0.18-0.90). On this ground, and despite OS is not the endpoint of the study, osimertinib might gain accelerated approval in upcoming years. However, before we are ready to adopt adjuvant osimertinib, further information regarding patient quality of life, response to subsequent lines of treatment, pattern (local/distant) and site of recurrence is needed.

CTONG-1104 demonstrated longer DFS and better toxicity profile with adjuvant gefitinib over A-Cht in completely resected N1/N2 EGFRmt NSCLC, highlighting their potential role to substitute $\mathrm{Cht}$ in this setting. However, the key endpoint of OS is negative. A closer look on the DFS curves for gefitinib vs. A-Cht, shows a separation in favour to gefitinib at 12 months but they come together again at 36 months which translates into similar OS. Despite the lack of survival advantage, the mOS of the gefitinib arm is one of the best reported for stage II-IIIA patients (2). This prolonged OS may be explained by the fact that gefitinib patients continue to respond to
EGFR-TKIs (osimertinib or other) at relapse. Molecular information from gefitinib-relapsed patients is not available but in SELECT study only 1 of the 15 patients that underwent a biopsy at relapse was T790M positive and $71 \%$ of patients were re-challenged with erlotinib (78). Therefore, patients who relapse after adjuvant gefitinib could be "rescued" with further TKI therapy. In fact, gefitinib patients treated with subsequent TKI had higher response rate and significantly longer survival after relapse compared to those receiving A-Cht followed by TKI or any other treatment. Moreover, survival after relapse was longer in patients treated for $\geq 18$ months with adjuvant gefitinib which may define a subgroup of patients specially sensitive to TKI therapy for whom EGFR inhibition given as a continuum (adjuvant and first line TKI) produces long survival. However, the continuum TKI strategy also entails some risks and a third of patients in either gefitinib or A-Cht arms did not received subsequent treatment at relapse. Notably, these considerations, are only hypothesis generating based in post hoc analyses with small numbers of patients.

At present, gefitinib could be considered as a potential Cht-free option for completely resected, stage II-IIIA N1/ N2 EGFRmt patients on the ground of longer DFS and better toxicity profile compared to A-Cht while maintaining TKI sensitivity at relapse. However, in light of the potential survival advantage of osimertinib in this setting, it is unlikely that gefitinib will be widely adopted. Osimertinib produces profound DFS benefit in stage II-IIIA patients and, based on subgroup analysis, the benefit is seen irrespective of previous $\mathrm{A}-\mathrm{Cht}$ and in node negative tumours $>4 \mathrm{~cm}$. Whether osimertinib could be licensed with promising but immature survival data remains an area of debate.

The apparent success of ADAURA over CTONG-1104 can be justified by the superiority of osimertinib as EGFR inhibitor. Notwithstanding this fact, there are other differences in trial design that might have impacted in patients' outcome. First, it could be argued that CTONG-1104 included patients with worse prognosis as A-Cht was not allowed and only stage II-IIA patients with node positive were eligible. Conversely, half of the patients in ADAURA had A-Cht and the primary endpoint analyses for DFS included stage II-IIIA patients irrespective of nodal status. However, these differences do not seem to affect the performance of the control groups which are comparable ( 2 and 3 years DFS were $44 \%$ and $28 \%$ vs. $40 \%$ and $27 \%$ in CTONG-1104 vs. ADAURA, respectively). 
Longer duration of EGFR therapy in ADAURA compared to CTONG-1104 (3 vs. 2 years) might have contributed to the benefit of osimertinib. In fact, the absolute DFS gain of gefitinib and osimertinib is relatively similar at 2 years $(30 \%$ and $45 \%)$ but the gap widens significantly at 3 years $(16 \%$ and $52 \%)$. Finally, is possible that patients treated with osimertinib had higher drug exposure. In ADAURA, $11 \%$ of patients discontinued permanently osimertinib due to toxicity. Despite this data is not available for CTONG-1104 for comparison, the DFS of gefitinib at 18 months was $\sim 80 \%$ but only $68 \%$ of the patients had $\geq 18$ months of gefitinib therapy. In FLAURA study, the treatment discontinuation rate for osimertinib was slightly lower compared to gefitinib/erlotinib (13\% vs. 18\%) (60). Allowing for all these considerations, the most relevant factor to explain the benefit of adjuvant osimertinb, over gefitinib is the fact that the former is a more potent EGFR inhibitor and this is line with results from the first line study FLAURA which demonstrated longer PFS and OS in patients treated with osimertinib compared to gefitinib/ erlotinib (61). The key to this advantage possibly lays in the mechanism of action of osimertinib which irreversibly and selectively targets both EGFR-TKI sensitising and EGFR T790M resistance mutations thus prolonging duration of response. Additionally, osimertinib offers better central nervous system (CNS) activity compared to first generation TKI. This not only has implications for patients diagnosed with CNS metastases but for the overall population at risk of developing CNS metastases. Notably, the frequency of CNS events in FLAURA was lower in patient receiving osimertinib although some asymptomatic patients could have been missed as brain imaging was not mandatory in patients without CNS metastases at baseline (60). Unfortunately, brain relapse is not and endpoint of ADUARA but any information in this subgroup of patients will be of great clinical value.

Another relevant question is how (neo)adjuvant TKI will compare with ICI in EGFR/ALK subgroup which classically have been excluded from ICI trials in advance disease as they were considered "cold tumours" and resistant to ICI (79). LCMC3 trial reported data on 7 EGFRmt and 1 ALK positive patients treated with neoadjuvant atezolizumab, 3/8 (38\%) patients achieved a pathological response of $40 \%$ to $50 \%$. At present, treatment strategies for advanced NSCLC are divided in two streams: tumours considered immunotherapy-sensitive and those oncogeneaddicted. Some of the ongoing trials evaluating ICI plus Cht will allow EGFR/ALK positive tumours and, despite small numbers, these data might challenge this paradigm in eNSCLC.

(Neo)adjuvant ICIs and TKIs could represent the next milestone for the treatment of completely resected eNSCLC. Previous (neo)adjuvant studies in EGFRmt gave a hint of TKIs activity in eNSCLC but ADAURA is the first trial to demonstrate a profound DFS improvement (and probable OS benefit) in stage I $>4 \mathrm{~cm}-$ IIIA. On this ground, osimertinib might gain accelerated approval in upcoming years. Beyond survival benefit, further information regarding pattern of recurrence, resistance mechanisms to osimertinib and quality of life are also key to effectively adopt this new regimen. These exciting results in EGFRmt population might spur a new era of (neo)adjuvant studies dedicated to other oncogene addicted subtypes thus bringing targeted therapy to early stage disease. Neoadjuvant ICI with/out platinum-based Cht produces high rates of MPR and pCR in small single arm studies. However, clinical research is still in its early days and ongoing phase III trials to validate the benefit of adding ICI to (neo)adjuvant Cht are awaited with great interest. Future challenges in this field include refining patients' selection, biomarkers, optimal treatment duration/sequencing and better understanding of immune-resistance.

In conclusion, targeted therapies and ICIs have definitely landed in eNSCLC, Hopefully, adequately powered and designed studies will be able to measure their true impact to improve survival rates.

\section{Acknowledgments}

Funding: None.

\section{Footnote}

Provenance and Peer Review: This article was commissioned by the Guest Editors (Silvia Novello, Francesco Passiglia) for the series "Looking for Chimeras in NSCLC: Widen Therapeutic Options Targeting Oncogenic Fusions" published in Translational Lung Cancer Research. The article has undergone external peer review.

Reporting Checklist: The authors have completed the Narrative Review reporting checklist. Available at http:// dx.doi.org/10.21037/tlcr-20-546

Peer Review File: Available at http://dx.doi.org/10.21037/ tlcr-20-546 
Conflicts of Interest: All authors have completed the ICMJE uniform disclosure form (available at http:// dx.doi.org/10.21037/tlcr-20-546). The series "Looking for Chimeras in NSCLC: Widen Therapeutic Options Targeting Oncogenic Fusions" was commissioned by the editorial office without any funding or sponsorship. MP serves as an unpaid editorial board member of Translational Lung Cancer Research from Sep 2019 to Sep 2021. Dr. VC reports personal fees from ROCHE, personal fees from BMS, personal fees from ASTRAZENECA, personal fees from BOEHRINGER, personal fees from MSD, outside the submitted work. Dr. MP reports advisory board for BMS, AstraZeneca, Roche and Takeda. Dr. RC reports personal fees from BMS, personal fees from Astrazeneca, personal fees from MSD, personal fees from Roche, outside the submitted work. The authors have no other conflicts of interest to declare.

Ethical Statement: The authors are accountable for all aspects of the work in ensuring that questions related to the accuracy or integrity of any part of the work are appropriately investigated and resolved.

Open Access Statement: This is an Open Access article distributed in accordance with the Creative Commons Attribution-NonCommercial-NoDerivs 4.0 International License (CC BY-NC-ND 4.0), which permits the noncommercial replication and distribution of the article with the strict proviso that no changes or edits are made and the original work is properly cited (including links to both the formal publication through the relevant DOI and the license). See: https://creativecommons.org/licenses/by-nc-nd/4.0/.

\section{References}

1. International Agency for Research on Cancer, World Health Organization. International Cancer Benchmarking Partnership (ICBP) SUVMARK-2. 2019. Available online: https://gco.iarc.fr/survival/survmark/ (Accessed 17th March 2020).

2. Goldstraw P, Crowley J, Chansky K, et al. The IASLC Lung Cancer Staging Project: proposals for the revision of the TNM stage groupings in the forthcoming (seventh) edition of the TNM Classification of malignant tumours. J Thorac Oncol 2007;2:706-14.

3. Postmus PE, Kerr KM, Oudkerk M, et al. Early and locally advanced non-small-cell lung cancer (NSCLC): ESMO Clinical Practice Guidelines for diagnosis, treatment and follow-up. Ann Oncol 2017;28:iv1-21.

4. Schil PE Van, Hellmann MD, Peters S, et al. ESMO Clinical Practice Guidelines for mNSCLC. Ann Oncol 2019;29:iv192-237.

5. Chemotherapy in non-small cell lung cancer: a metaanalysis using updated data on individual patients from 52 randomised clinical trials. Non-small Cell Lung Cancer Collaborative Group. BMJ 1995;311:899-909.

6. Strauss GM, Herndon JE, Maddaus MA, et al. Adjuvant paclitaxel plus carboplatin compared with observation in stage IB non-small-cell lung cancer: CALGB 9633 With the Cancer and Leukemia Group B, Radiation Therapy Oncology Group, and North Central Cancer Treatment Group Study Groups. J Clin Oncol 2008;26:5043-51.

7. Artal Cortés Á, Calera Urquizu L, Hernando Cubero J. Adjuvant chemotherapy in non-small cell lung cancer: state-of-the-art. Transl Lung Cancer Res 2015;4:191-7.

8. Pignon JP, Tribodet H, Scagliotti GV, et al. Lung adjuvant cisplatin evaluation: a pooled analysis by the LACE Collaborative Group. J Clin Oncol 2008;26:3552-9.

9. Douillard JY, Rosell R, De Lena M, et al. Adjuvant vinorelbine plus cisplatin versus observation in patients with completely resected stage IB-IIIA non-small-cell lung cancer (Adjuvant Navelbine International Trialist Association [ANITA]): a randomised controlled trial. Lancet Oncol 2006;7:719-27.

10. Butts CA, Ding K, Seymour L, et al. Randomized phase III trial of vinorelbine plus cisplatin compared with observation in completely resected stage IB and II nonsmall-cell lung cancer: updated survival analysis of JBR-10. J Clin Oncol 2010;28:29-34.

11. Douillard JY, Tribodet H, Aubert D, et al. Adjuvant cisplatin and vinorelbine for completely resected non-small cell lung cancer: subgroup analysis of the Lung Adjuvant Cisplatin Evaluation. J Thorac Oncol 2010;5:220-8.

12. Gilligan D, Nicolson M, Smith I, et al. Preoperative chemotherapy in patients with resectable non-small cell lung cancer: results of the MRC LU22/NVALT 2/ EORTC 08012 multicentre randomised trial and update of systematic review. Lancet 2007;369:1929-37.

13. NSCLC Meta-analysis Collaborative Group. Preoperative chemotherapy for non-small-cell lung cancer: a systematic review and meta-analysis of individual participant data. Lancet 2014;383:1561-71.

14. Burdett SS, Stewart LA, Rydzewska L. Chemotherapy and surgery versus surgery alone in non-small cell lung cancer. Cochrane Database Syst Rev 2007;(3):CD006157.

15. Felip E, Rosell R, Maestre JA, et al. Preoperative 
chemotherapy plus surgery versus surgery plus adjuvant chemotherapy versus surgery alone in early-stage nonsmall-cell lung cancer. J Clin Oncol 2010;28:3138-45.

16. Lim E, Harris G, Patel A, et al. Preoperative versus postoperative chemotherapy in patients with resectable non-small cell lung cancer: systematic review and indirect comparison meta-analysis of randomized trials. J Thorac Oncol 2009;4:1380-8.

17. Hellmann MD, Chaft JE, William WN, et al. Pathological response after neoadjuvant chemotherapy in resectable non-small-cell lung cancers: proposal for the use of major pathological response as a surrogate endpoint. Lancet Oncol 2014;15:e42-50.

18. Betticher DC, Hsu Schmitz SF, Tötsch M, et al. Prognostic factors affecting long-term outcomes in patients with resected stage IIIA pN2 non-small-cell lung cancer: 5-year follow-up of a phase II study. Br J Cancer 2006;94:1099-106.

19. Depierre A, Milleron B, Moro-Sibilot D, et al. Preoperative chemotherapy followed by surgery compared with primary surgery in resectable stage I (except T1N0), II, and IIIa non-small-cell lung cancer. J Clin Oncol 2002;20:247-53.

20. Mouillet G, Monnet E, Milleron B, et al. Pathologic complete response to preoperative chemotherapy predicts cure in early-stage non-small-cell lung cancer: combined analysis of two IFCT randomized trials. J Thorac Oncol 2012;7:841-9.

21. Junker K, Langner K, Klinke F, et al. Grading of tumour regression in non-small cell lung cancer: morphology and prognosis. Chest 2001;120:1584-91.

22. Pataer A, Kalhor N, Correa AM, et al. Histopathologic response criteria predict survival of patients with resected lung cancer after neoadjuvant chemotherapy. J Thorac Oncol 2012;7:825-32.

23. Cottrell TR, Thompson ED, Forde PM, et al. Pathologic features of response to neoadjuvant anti-PD-1 in resected non-small-cell lung carcinoma: a proposal for quantitative immune-related pathologic response criteria (irPRC). Ann Oncol 2018;29:1853-60.

24. Reck M, Rodríguez-Abreu D, Robinson AG, et al. Pembrolizumab versus chemotherapy for PD-L1positive non-small-cell lung cancer. N Engl J Med 2016;375:1823-33

25. Paz-Ares L, Luft A, Vicente D, et al. Pembrolizumab plus chemotherapy for squamous non-small-cell lung cancer. $\mathrm{N}$ Engl J Med 2018;379:2040-51.

26. Gandhi L, Rodríguez-Abreu D, Gadgeel S, et al.
Pembrolizumab plus chemotherapy in metastatic nonsmall-cell lung cancer. N Engl J Med 2018;378:2078-92.

27. Socinski MA, Jotte RM, Cappuzzo F, et al. Atezolizumab for first-line treatment of metastatic nonsquamous NSCLC. N Engl J Med 2018;378:2288-301.

28. Hellmann MD, Paz-Ares L, Bernabe Caro R, et al. Nivolumab plus ipilimumab in advanced non-small-cell lung cancer. N Engl J Med 2019;381:2020-31.

29. Reck M, Ciuleanu TE, Dols MC, et al. Nivolumab (NIVO) + ipilimumab (IPI) + 2 cycles of platinum-doublet chemotherapy (chemo) vs 4 cycles chemo as first-line (1L) treatment (tx) for stage IV/recurrent non-small cell lung cancer (NSCLC): CheckMate 9LA. J Clin Oncol 2020;38:abstr 9501.

30. Addeo A, Banna GL, Metro G, et al. Chemotherapy in combination with immune checkpoint inhibitors for the first-line treatment of patients with advanced non-small cell lung cancer: a systematic review and literature-based meta-analysis. Front Oncol 2019;9:264.

31. Rittmeyer A, Barlesi F, Waterkamp D, et al. Atezolizumab versus docetaxel in patients with previously treated non-small-cell lung cancer (OAK): a phase 3, openlabel, multicentre randomised controlled trial. Lancet 2017;389:255-65.

32. Borghaei H, Paz-Ares L, Horn L, et al. Nivolumab versus docetaxel in advanced nonsquamous non-small-cell lung cancer. N Engl J Med 2015;373:1627-39.

33. Brahmer J, Reckamp KL, Baas P, et al. Nivolumab versus docetaxel in advanced squamous-cell non-small-cell lung cancer. N Engl J Med 2015;373:123-35.

34. Herbst RS, Baas P, Kim DW, et al. Pembrolizumab versus docetaxel for previously treated, PD-L1-positive, advanced non-small-cell lung cancer (KEYNOTE-010): a randomised controlled trial. Lancet 2016;387:1540-50.

35. Antonia SJ, Villegas A, Daniel D, et al. Durvalumab after chemoradiotherapy in stage III non-small-cell lung cancer. N Engl J Med 2017;377:1919-29.

36. Antonia SJ, Borghaei H, Ramalingam SS, et al. Four-year survival with nivolumab in patients with previously treated advanced non-small-cell lung cancer: a pooled analysis. Lancet Oncol 2019;20:1395-408.

37. Gray JE, Villegas A, Daniel D, et al. Three-year overall survival with durvalumab after chemoradiotherapy in stage III NSCLC-update from PACIFIC. J Thorac Oncol 2020;15:288-93.

38. Eggermont AMM, Chiarion-sileni V, Grob JJ, et al. Prolonged survival in stage III melanoma with ipilimumab adjuvant therapy. N Engl J Med 2016;375:1845-55. 
39. Forde PM, Chaft JE, Smith KN, Aet al. Neoadjuvant PD-1 blockade in resectable lung cancer. $\mathrm{N}$ Engl J Med 2018;378:1976-86.

40. Kwiatkowski DJ Rusch VW, Chaft JE, et al. Neoadjuvant atezolizumab in resectable non-small cell lung cancer (NSCLC): interim analysis and biomarker data from a multicenter study (LCMC3). J Clin Oncol 2019;37:8503.

41. Gao S, Li N, Gao S, et al. Neoadjuvant PD-1 inhibitor (Sintilimab) in NSCLC. J Thorac Oncol 2020;15:816-26.

42. Cascone T, William WN, Weissferdt A, et al. Neoadjuvant nivolumab $(\mathrm{N})$ or nivolumab plus ipilimumab (NI) for resectable non-small cell lung cancer (NSCLC): Clinical and correlative esults from the NEOSTAR study. J Clin Oncol 2019;37:8504.

43. Provencio M, Nadal E, Insa A, et al. Neoadjuvant chemoimmunotherapy for the treatment of stage IIIA resectable non-small-cell lung cancer (NSCLC): A phase II multicenter exploratory study-Final data of patients who underwent surgical assessment. J Clin Oncol 2019;37:8509.

44. Shu CA, Grigg C, Chiuzan C, et al. Neoadjuvant atezolizumab + chemotherapy in resectable non-small cell lung cancer (NSCLC). J Clin Oncol 2018;36:8532.

45. Reuss JE, Smith KN, Anagnostou V, et al. Neoadjuvant nivolumab in resectable non-small cell lung cancer: extended follow-up and molecular markers of response. J Clin Oncol 2019;37:8524.

46. Wu YL, Zhou C, Hu CP, et al. Afatinib versus cisplatin plus gemcitabine for first-line treatment of Asian patients with advanced non-small-cell lung cancer harbouring EGFR mutations (LUX-Lung 6): an open-label, randomised phase 3 trial. Lancet Oncol 2014;15:213-22.

47. Solomon BJ, Mok T, Kim DW, et al. First-line crizotinib versus chemotherapy in ALK-positive lung cancer. N Engl J Med 2014;371:2167-77.

48. Rosell R, Carcereny E, Gervais R, et al. Erlotinib versus standard chemotherapy as first-line treatment for European patients with advanced EGFR mutation-positive non-small-cell lung cancer (EURTAC): a multicentre, open-label, randomised phase 3 trial. Lancet Oncol 2012;13:239-46.

49. He Q, Xin P, Zhang M, et al. The impact of epidermal growth factor receptor mutations on the prognosis of resected non-small cell lung cancer: a meta-analysis of literatures. Transl Lung Cancer Res 2019;8:124-34.

50. D'Angelo SP, Janjigian YY, Ahye N, et al. Distinct clinical course of EGFR-mutant resected lung cancers: results of testing of 1118 surgical specimens and effects of adjuvant gefitinib and erlotinib. J Thorac Oncol 2012;7:1815-22.
51. Paik JH, Choi CM, Kim H, et al. Clinicopathologic implication of ALK rearrangement in surgically resected lung cancer: a proposal of diagnostic algorithm for ALKrearranged adenocarcinoma. Lung Cancer 2012;76:403-9.

52. Pan Y, Zhang Y, Li Y, et al. ALK, ROS1 and RET fusions in 1139 lung adenocarcinomas: a comprehensive study of common and fusion pattern-specific clinicopathologic, histologic and cytologic features. Lung Cancer 2014;84:121-6.

53. Ahn HK, Choi YL, Han JH, et al. Epidermal growth factor receptor mutation and treatment outcome of mediastinoscopic N2 positive non-small cell lung cancer patients treated with neoadjuvant chemoradiotherapy followed by surgery. Lung Cancer 2013;79:300-6.

54. Rizvi NA, Rusch V, Pao W, et al. Molecular characteristics predict clinical outcomes: prospective trial correlating response to the EGFR tyrosine kinase inhibitor gefitinib with the presence of sensitizing mutations in the tyrosine binding domain of the EGFR gene. Clin Cancer Res 2011;17:3500-6.

55. Han J, Xiong L, Sun J, et al. Erlotinib as neoadjuvant treatment in endobronchial ultrasound confirmed stage IIIA-N2 non-small cell lung cancer (NSCLC) patients with epidermal growth factor receptor (EGFR) mutation (EXON 19 OR 21) (NCT01217619, ESTERN): a prospective, single arm, phase II clinical trial. Ann Oncol 2015;26:124-8.

56. Zhong W, Yang X, Yan H, et al. Phase II study of biomarker-guided neoadjuvant treatment strategy for IIIA-N2 non-small cell lung cancer based on epidermal growth factor receptor mutation status. J Hematol Oncol 2015;8:54.

57. Zhong WZ, Chen KN, Chen C, et al. Erlotinib versus gemcitabine plus cisplatin as neoadjuvant treatment of stage IIIA-N2 EGFR-mutant non-small-cell lung cancer (EMERGING-CTONG 1103): a randomized phase II study. J Clin Oncol 2019;37:2235-45.

58. Zhang C, Li SL, Nie Q, et al. Neoadjuvant crizotinib in resectable locally advanced non-small cell lung cancer with ALK rearrangement. J Thorac Oncol 2019;14:726-31.

59. Chaft JE, Oxnard GR, Sima CS, et al. Disease flare after tyrosine kinase inhibitor discontinuation in patients with EGFR-mutant lung cancer and acquired resistance to erlotinib or gefitinib: implications for clinical trial design. Clin Cancer Res 2011;17:6298-303.

60. Soria JC, Ohe Y, Vansteenkiste J, et al. Osimertinib in untreated EGFR-mutated advanced non-small-cell lung cancer. N Engl J Med 2018;378:113-25. 
61. Ramalingam SS, Gray JE, Ohe Y, et al. Osimertinib vs comparator EGFR-TKI as first-line treatment for EGFRm advanced NSCLC (FLAURA): final overall survival analysis. Ann Oncol 2019;30:V914-5.

62. Goss GD, O'Callaghan C, Lorimer I, et al. Gefitinib versus placebo in completely resected non-small-cell lung cancer: results of the NCIC CTG BR19 study. J Clin Oncol 2013;31:3320-6.

63. Kelly K, Altorki NK, Eberhardt WEE, et al. Adjuvant erlotinib versus placebo in patients with stage IBIIIA nonsmall-cell lung cancer (RADIANT): A randomized, double-blind, Phase III trial. J Clin Oncol 2015;33:4007-14.

64. Pennell NA, Neal JW, Chaft JE, et al. SELECT: a phase II trial of adjuvant erlotinib in patients with resected epidermal growth factor receptor-mutant non-small-cell lung cancer. J Clin Oncol 2019;37:97-104.

65. Li N, Ou W, Ye X, et al. Pemetrexed-carboplatin adjuvant chemotherapy with or without gefitinib in resected stage IIIA-N2 non-small cell lung cancer harbouring EGFR mutations: a randomized, phase II study. Ann Surg Oncol 2014;21:2091-6.

66. Feng S, Wang Y, Cai K, et al. Randomized adjuvant chemotherapy of EGFR-mutated non-small cell lung cancer patients with or without icotinib consolidation therapy. PLoS One 2015;10: e0140794.

67. Herbst RS, Tsuboi M, John T, et al. Osimertinib as adjuvant therapy in patients (pts) with stage IB-IIIA EGFR mutation positive (EGFRm) NSCLC after complete tumortumour resection: ADAURA. J Clin Oncol 2020;38:LBA5.

68. Zhong WZ, Wang Q, Mao WM, et al. Gefitinib versus vinorelbine plus cisplatin as adjuvant treatment for stage II-IIIA (N1-N2) EGFR-mutant NSCLC (ADJUVANT/ CTONG1104): a randomised, open-label, phase 3 study. Lancet Oncol 2018;19:139-48.

69. Wu YL, Zhong W, Wang Q, et al. CTONG1104: Adjuvant gefitinib versus chemotherapy for resected N1N2 NSCLC with EGFR mutation-final overall survival analysis of the randomized phase III trial 1 analysis of the randomized phase III trial. J Clin Oncol 2020;38:abstr 9005.

70. Yue D, Xu S, Wang Q, et al. Erlotinib versus vinorelbine plus cisplatin as adjuvant therapy in Chinese patients with stage IIIA EGFR mutation-positive non-small-cell lung cancer (EVAN): a randomised, open-label, phase 2 trial. Lancet Respir Med 2018;6:863-73.

71. Kelly K, Chansky K, Gaspar LE, et al. Phase III trial of maintenance gefitinib or placebo after concurrent chemoradiotherapy and docetaxel consolidation in inoperable stage III non-small-cell lung cancer: SWOG S0023. J Clin Oncol 2008;26:2450-6.

72. Thatcher N, Chang A, Parikh P, et al. Gefitinib plus best supportive care in previously treated patients with refractory advanced non-small-cell lung cancer: results from a randomised, placebo-controlled, multicentre study (Iressa Survival Evaluation in Lung Cancer). Lancet 2005;366:1527-37.

73. Huang Q, Li J, Sun Y, et al. Efficacy of EGFR tyrosine kinase inhibitors in the adjuvant treatment for operable non-small cell lung cancer by a meta-analysis. Chest 2016;149:1384-92.

74. Eberhardt WE, Pöttgen C, Gauler TC, et al. Phase III study of surgery versus definitive concurrent chemoradiotherapy boost in patients with resectable stage IIIA(N2) and selected IIIB non-small-cell lung cancer after induction chemotherapy and concurrent chemoradiotherapy (ESPATUE). J Clin Oncol 2015;33:4194-201.

75. Albain KS, Swann RS, Rusch VW, et al. Radiotherapy plus chemotherapy with or without surgical resection for stage III non-small-cell lung cancer: a phase III randomised controlled trial. Lancet 2009;374:379-86.

76. Liu J, Blake SJ, Yong MC, et al. Improved efficacy of neoadjuvant compared to adjuvant immunotherapy to eradicate metastatic disease. Cancer Discov 2016;6:1382-99.

77. Tazza M, Metro G. Adjuvant treatment of non-small cell lung cancer: Focus on targeted therapy. J Thorac Dis 2017;9:4064-9.

78. Zhai H, Zhong W, Yang X, et al. Neoadjuvant and adjuvant epidermal growth factor receptor tyrosine kinase inhibitor (EGFR-TKI) therapy for lung cancer. Transl Lung Cancer Res 2015;4:82-93.

79. Soo RA, Lim SM, Syn NL, et al. Immune checkpoint inhibitors in epidermal growth factor receptor mutant non-small cell lung cancer: current controversies and future directions. Lung Cancer 2018;115:12-20.

Cite this article as: Ortega-Franco A, Calvo V, Franco F, Provencio M, Califano R. Integrating immune checkpoint inhibitors and targeted therapies in the treatment of early stage non-small cell lung cancer: a narrative review. Transl Lung Cancer Res 2020;9(6):2656-2673. doi: 10.21037/tlcr-20-546 